\title{
Symmetry energy at subnuclear densities and nuclei in a neutron-star crust
}

\section{Kazuhiro Oyamatsu*}

Department of Media Theories and Production, Aichi Shukutoku University, Nagakute-cho, Aichi, 480-1197, Japan

E-mail: oyakeasu.aasa.ac.jp

\section{Kei lida}

Faculty of Science, Kochi University, Kochi 780-8520, Japan

E-mail: iida@cc.kochi-u.ac.jp

\begin{abstract}
We examine how the properties of inhomogeneous nuclear matter at subnuclear densities depend on the density dependence of the symmetry energy. We focus on a parameter that characterizes the density dependence of the symmetry energy $S(n)$ at about nuclear density $n_{0}$. It is the density symmetry coefficient $L=3 n_{0}(d S / d n)_{n=n_{0}}$. From the saturation properties of stable nuclei, we find that the usual symmetry energy coefficient $S_{0}=S\left(n_{0}\right)$ has a strong correlation with $L$ $\left(S_{0} \approx 28+0.075 L \mathrm{MeV}\right)$. Thus, we have essentially only one uncertain parameter $L$. Using a macroscopic nuclear model we calculate the sizes and shapes of nuclei in neutron star matter at zero temperature for different assumptions about the density dependence of the symmetry energy. We find that for smaller symmetry energy at subnuclear densities, corresponding to larger density symmetry coefficient $L$, the charge number of nuclei is smaller, and the critical density at which matter with nuclei or bubbles becomes uniform is lower. The value of the key parameter $L$ could be constrained from global neutron-excess dependencies of radii and masses in nuclear chart. Hence, systematic measurements of radii and masses of neutron-rich nuclei could help reveal the structure of matter around the inner boundary of a neutron-star crust.
\end{abstract}

10th Symposium on Nuclei in the Cosmos

July 27 - August 12008

Mackinac Island, Michigan, USA

\footnotetext{
* Speaker.
} 


\section{Introduction}

The macroscopic properties of nuclei, such as masses and radii, are dominated by the equation of state (EOS) of nuclear matter. The EOS is relatively well-known for symmetric nuclear matter thanks to empirical masses and radii of stable nuclei. Nuclei in neutron-star crusts are extremely neutron-rich. Their macroscopic properties are dominated by the EOS of asymmetric nuclear matter, which is poorly known at present. In this study, we systematically examine which of the following five EOS parameters most significantly controls the macroscopic properties of neutron-rich nuclei in laboratories and in neutron-star crusts. The first three EOS parameters are the saturation energy, $w_{0}$, the saturation density, $n_{0}$, and the incompressibility, $K_{0}$, of symmetric nuclear matter. The remaining two parameters $L$ and $S_{0}$ are associated with the density dependent symmetry energy $S(n): S_{0}=S(n)$ is the symmetry energy at $n=n_{0}$, and $L=3 n_{0}(d S / d n)_{n=n_{0}}$ is the symmetry energy density derivative coefficient.

\section{Family of empirical EOS}

We adopt a macroscopic nuclear model which can reasonably describe not only stable and unstable nuclei in laboratories [1] but also extremely neutron-rich nuclei in neutron-star crusts [2]. In this model, the mass of a nuclide is given by

$$
\int d^{3} r \varepsilon\left(n_{n}(\mathbf{r}), n_{p}(\mathbf{r})\right)+F_{0} \int d^{3} r|\nabla n(\mathbf{r})|^{2}+\frac{e^{2}}{2} \int d^{3} r \int d^{3} r^{\prime} \frac{n_{p}(\mathbf{r}) n_{p}\left(\mathbf{r}^{\prime}\right)}{\left|\mathbf{r}-\mathbf{r}^{\prime}\right|}+m_{n} N+\left(m_{p}+m_{e}\right) Z .
$$

Here $\varepsilon\left(n_{n}, n_{p}\right)$ is the energy density (the EOS) as a function of the neutron (proton) density $n_{n}\left(n_{p}\right)$, $F_{0}$ is a surface energy parameter, and the third term is the Coulomb energy. Note that the integration in the first term over matter in the vicinity of the nuclear surface also contributes about half of the surface energy. The detailed description of the model including the parameterization of the EOS is given in Ref. [1].

We systematically generate about 200 sets of the EOS $\varepsilon$ and the constant $F_{0}$, which reproduce almost equally well masses and radii of stable nuclei [1]. Figure 1 shows two important EOS properties obtained from stable nuclei. There is a strong correlation between $S_{0}$ and $L$. The values of $L$ and $K_{0}$ can not be constrained well from masses and radii of stable nuclei. From the nine representative EOS's in Fig. 2, one can verify that the family of EOS's included in this study spans much of range spanned by phenomenological EOS's of contemporary use.

\section{Neutron-rich nuclei in laboratories}

We calculate masses and radii of neutron-rich nuclei using the family of the EOS's to examine the EOS dependence. As shown in Fig. 3, masses and radii of neutron-rich nuclei in laboratories have appreciable sensitivities to the $L$ value. This could be useful to obtain empirical constraint of the $L$ value. The dependence on the $K_{0}$ value is comparatively weak.

\section{Nuclei in neutron-star crusts}

Nuclei in neutron-star crusts are also calculated using this family of the EOS's [3]. Figure 4 shows the proton number $Z$ of nuclei and the average proton fraction of matter in the inner crust. 

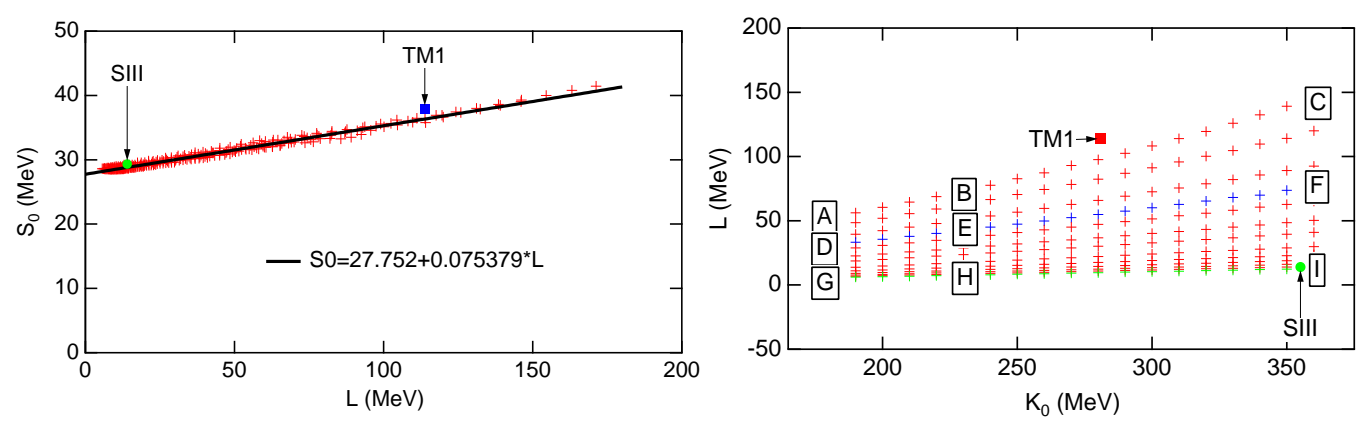

Figure 1: The sets of $\left(L, S_{0}\right)$ (left panel) and $\left(K_{0}, L\right)$ (right panel) consistent with the mass and radius data for stable nuclei. The labels A-I denote the sets for which we perform detailed calculations of the ground state properties of inhomogeneous nuclear matter at subnuclear densities in Fig. 4. For comparison, the values calculated from two mean-field models [TM1 (square) and SIII (dot)], which are known to be extreme cases [1], are plotted. The plot shows that our sets of $\left(L, K_{0}\right)$ effectively cover such extreme cases.

The larger $L$, the smaller $Z$ and $Y_{p}$. This reflects the fact that the the symmetry energy $S(n)$ at subnuclear densities is smaller for larger $L$.

We further examine the structure of the inner boundary of the neutron-star crust. Specifically, we calculate the core-crust boundary density and the density where the spherical nuclei become unstable against nuclear fission. As shown in Fig. 5, the former density is a decreasing function of $L$ almost independently of $K_{0}$ while the latter is nearly constant. Here, the $K_{0}$ dependence is mainly reflected in the widths of the dependences of these two quantities on $L$. It is noted that the density region between the two densities correspond to the region for pasta nuclei. Hence, the existence of pasta nuclei in a neutron-star crust is determined by the $L$ value. If $L \lesssim 100 \mathrm{MeV}$, pasta nuclei exist in a neutron-star crust.

\section{Conclusions}

The values of $L$ and $K_{0}$ are constrained poorly from masses and radii of stable nuclei. However,radii and masses of neutron-rich nuclei have appreciable sensitivity to the $L$ value. As for neutron-star inner crusts, proton numbers of nuclei and average proton fractions in the matter decrease with $L$. The core-crust boundary density of neutron star is also dependent on $L$. The existence of the pasta phase is determined by $L$. The pasta phase exists in a neutron-star crust if $L \lesssim 100 \mathrm{MeV}$. It is quite interesting that the above nuclear properties are almost independent of $K_{0}$. However, the present uncertainty in $L$ is too large; better constraints on $L$ are needed. Systematic experimental studies of nuclear masses and sizes of unstable nuclei in laboratories will help determine the $L$ value and the existence of pasta nuclei in neutron stars.

\section{References}

[1] K. Oyamatsu and K. Iida, Prog. Theor. Phys. 109 (2003) 631.

[2] K. Oyamatsu, Nucl. Phy. A561 (1993) 431.

[3] K. Oyamatsu and K. Iida, Phys. Rev. C 75 (2007) 015801. 


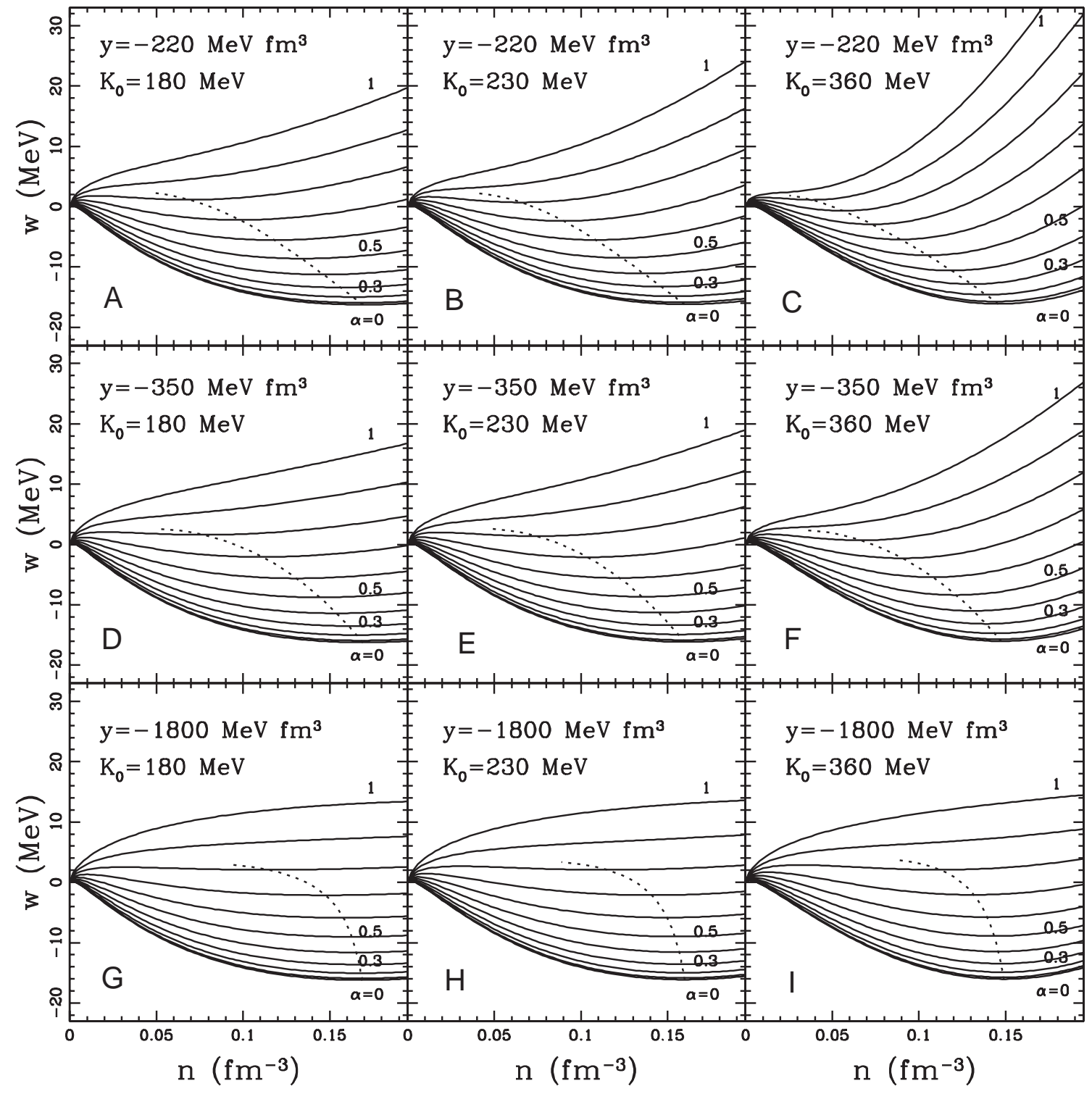

Figure 2: Energy per nucleon of nuclear matter for the nine sets of $\left(L, K_{0}\right)$ referred to as A-I in Fig. 1. In each panel, the solid lines are the energy at neutron excess $\alpha=0,0.3,0.5,0.8,1$. The dotted lines show the saturation lines joining the saturation points of asymmetric nuclear matter. The symbol $y$ denotes the slope of the saturation line at $\alpha=0$. 

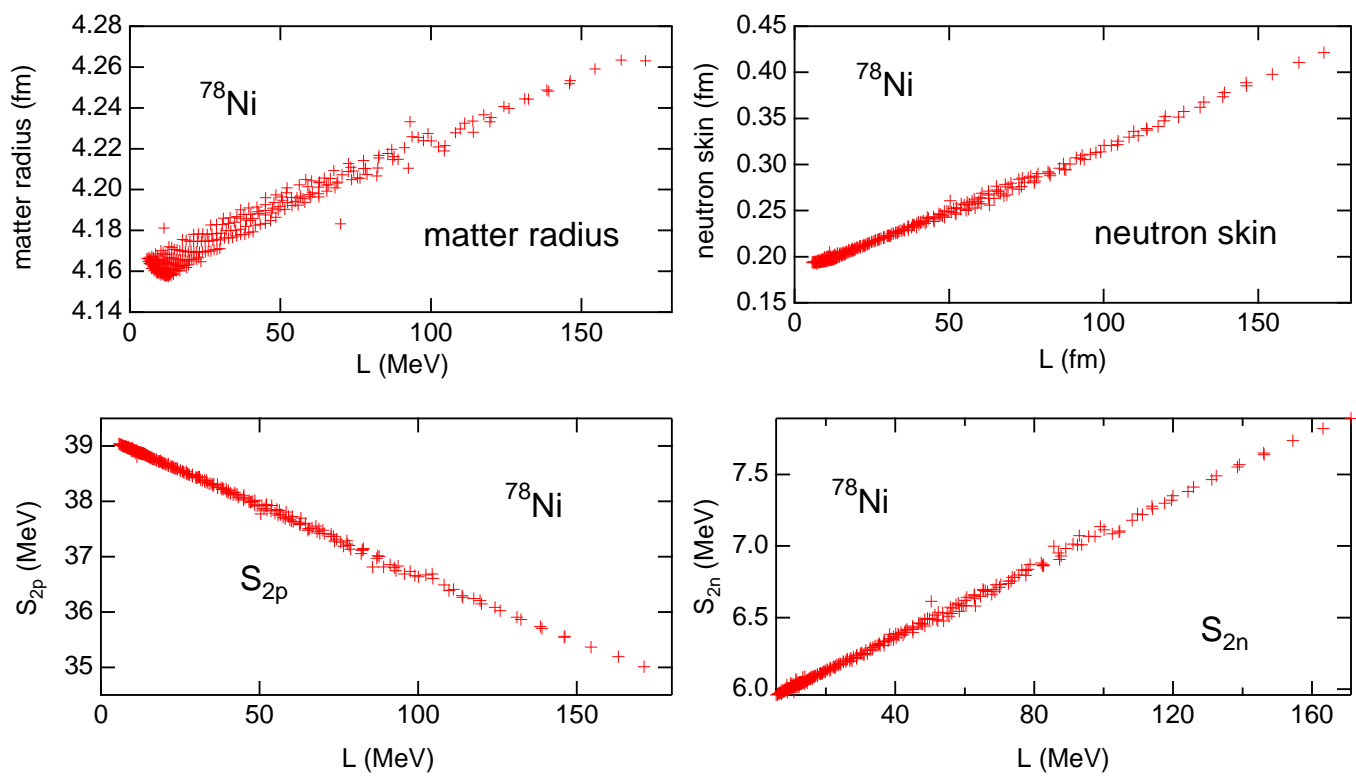

Figure 3: Matter radius, neutron skin, two proton separation energy and two neutron separation energy of ${ }^{78} \mathrm{Ni}$ caluated with the family of EOS's.
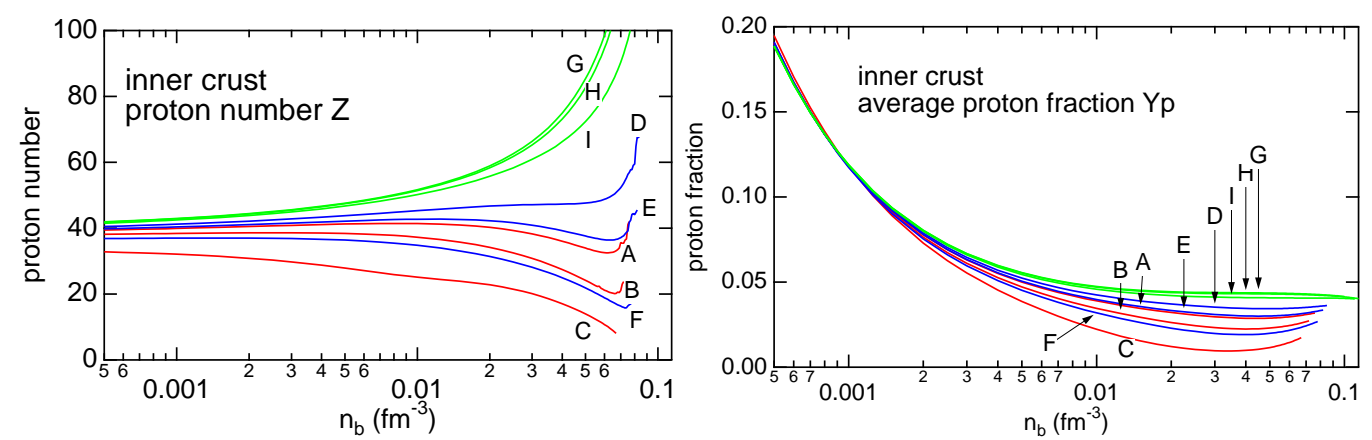

Figure 4: The proton number $Z$ of nuclei and average proton fraction $Y_{p}$ of the matter in the inner crust of a neutron star for EOS A-I in Fig. 2.

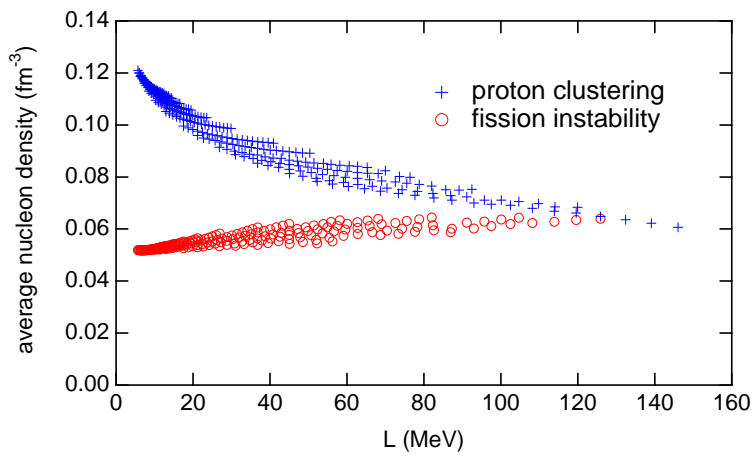

Figure 5: The onset density of proton clustering in uniform nuclear matter as a function of $L$ (crosses). For comparison, we plot the density where spherical nuclei become unstable against nuclear fission(circles). 